\title{
Mn-doped Ga(As,P) and (Al,Ga)As ferromagnetic semiconductors: Electronic structure calculations
}

\author{
J. Mašek, ${ }^{1}$ J. Kudrnovský, ${ }^{1}$ F. Máca, ${ }^{1}$ Jairo Sinova, ${ }^{2}$ A. H. MacDonald, ${ }^{3}$ R. P. Campion, ${ }^{4}$ \\ B. L. Gallagher, ${ }^{4}$ and T. Jungwirth ${ }^{5,4}$ \\ ${ }_{1}^{1}$ Institute of Physics ASCR, Na Slovance 2, 18221 Praha 8, Czech Republic \\ ${ }^{2}$ Department of Physics, Texas A\&M University, College Station, Texas 77843-4242, USA \\ ${ }^{3}$ Department of Physics, University of Texas at Austin, Austin, Texas 78712-1081, USA \\ ${ }^{4}$ School of Physics and Astronomy, University of Nottingham, Nottingham NG7 2RD, United Kingdom \\ ${ }^{5}$ Institute of Physics ASCR, Cukrovarnická 10, 16253 Praha 6, Czech Republic
}

(Received 6 September 2006; published 12 January 2007)

\begin{abstract}
A remarkable progress towards functional ferromagnetic semiconductor materials for spintronics has been achieved in p-type $(\mathrm{Ga}, \mathrm{Mn})$ As. Robust hole-mediated ferromagnetism has, however, been observed also in other III-V hosts such as antimonides, $\mathrm{GaP}$, or $(\mathrm{Al}, \mathrm{Ga}) \mathrm{As}$, which opens a wide area of possibilities for optimizing the host composition towards higher ferromagnetic Curie temperatures. Here we explore theoretically hole-mediated ferromagnetism and $\mathrm{Mn}$ incorporation in $\mathrm{Ga}(\mathrm{As}, \mathrm{P})$ and $(\mathrm{Al}, \mathrm{Ga}) \mathrm{As}$ ternary hosts. While alloying $(\mathrm{Ga}, \mathrm{Mn}) \mathrm{As}$ with $\mathrm{Al}$ has only a small effect on the Curie temperature we predict a sizable enhancement of Curie temperatures in the smaller lattice constant $\mathrm{Ga}(\mathrm{As}, \mathrm{P})$ hosts. Mn-doped $\mathrm{Ga}(\mathrm{As}, \mathrm{P})$ is also favorable, as compared to $(\mathrm{Al}, \mathrm{Ga}) \mathrm{As}$, with respect to the formation of carrier and moment compensating interstitial $\mathrm{Mn}$ impurities. In $(\mathrm{Ga}, \mathrm{Mn})(\mathrm{As}, \mathrm{P})$ we find a marked decrease of the partial concentration of these detrimental impurities with increasing $\mathrm{P}$ content.
\end{abstract}

DOI: 10.1103/PhysRevB.75.045202

PACS number(s): 75.50.Pp, 75.30.Hx, 73.61.Ey

\section{INTRODUCTION}

Ordered by increasing band gap and decreasing lattice constant of the III-V host, electronic states associated with $\sim 1-10 \%$ of substitutional $\mathrm{Mn}_{\mathrm{III}}$ impurities may fall in the following three qualitative categories: ${ }^{1-3}$ (i) The main peak in the partial density of states of the majority-spin $\mathrm{Mn} d^{5}$ electrons is well below the Fermi energy and these states form a local moment close to 5 Bohr magnetons. Mn acts here as an acceptor and the delocalized holes have a character of the host states near the top of the valence band with a small admixture of the Mn $d$-orbital weight. In these metallic (III,Mn)V ferromagnets the coupling between Mn local moments is mediated by delocalized band holes via the kineticexchange mechanism. ${ }^{4-8}$ (ii) The second regime, often referred to as a double-exchange ferromagnet, is characterized by a stronger hybridization of Mn $d$ states near the Fermi energy and by holes occupying an impurity band which is detached from the host semiconductor valence band. In this picture electrical conduction and Mn-Mn exchange coupling are both realized through hopping within the impurity band. ${ }^{9-17}$ (iii) Finally, the substitutional $\mathrm{Mn}_{\mathrm{III}}$ impurities may undergo a transition from a divalent $\left(d^{5}\right)$ acceptor to a trivalent $\left(d^{4}\right)$ neutral state. This strongly correlated $d^{4}$ center, with four occupied $d$ orbitals and a nondegenerate empty $t_{2 g} d$ level shifted deep into the host band gap, may form as a result of a spontaneous (Jahn-Teller) lowering of the cubic symmetry near the Mn site. Systems with dominant $d^{4}$ character of Mn impurities, reminiscent of a charge transfer insulator, will inevitably require additional charge codoping to provide for ferromagnetic coupling between dilute Mn moments. . $^{2,3,17}$

The internal reference rule, ${ }^{18,19}$ which states that energy levels derived from $\mathrm{Mn}$ are constant across semiconductor compound families with properly aligned bands, serves as useful guidance for associating individual III-V hosts with one of the three categories listed above. As seen from Fig. 1, Mn-doped InSb, InAs, and GaAs can be expected to exhibit long range $\mathrm{Mn}-\mathrm{Mn}$ coupling mediated by delocalized holes in the host valence band. This picture has indeed been corroborated by a number of experimental studies. ${ }^{1}$ Measured Curie temperatures, currently ranging from $7 \mathrm{~K}$ in the narrow gap, large lattice constant (In,Mn)Sb (Ref. 20) to $173 \mathrm{~K}$ in the wider gap smaller, lattice constant $(\mathrm{Ga}, \mathrm{Mn}) \mathrm{As},{ }^{21,22}$ are consistent with the kinetic-exchange model.

On the opposite side of the spectrum of III-V hosts, bulk $(\mathrm{Ga}, \mathrm{Mn}) \mathrm{N}$ is an example of a wide gap material in which $\mathrm{Mn}$ does not provide for a significant hole doping. ${ }^{23,24}$ Considering the width of the band gap only, GaP would fall in the same category as GaN. As shown in Fig. 1, however, the bands are significantly offset to higher energies in GaP and

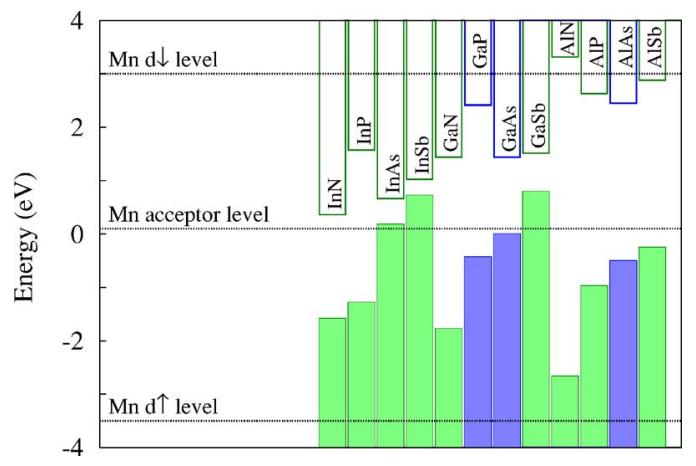

FIG. 1. (Color online) Valence band and conduction band offsets across the family of III-V semiconductors. Mn derived energy levels are constant in this diagram according to the empirical internal reference rule. 
the resulting Mn acceptor level is shallow enough to lead to a robust hole mediated ferromagnetism at $\mathrm{Mn}$ dopings of several percent. Experiments in $(\mathrm{Ga}, \mathrm{Mn}) \mathrm{P}$ reported to date suggest $\mathrm{t}^{25}$ the presence of an impurity band in this III-V host, although more studies are needed to establish whether this character is intrinsic to $(\mathrm{Ga}, \mathrm{Mn}) \mathrm{P}$ or occurs partly due to other unintentional impurities present in the studied systems. $^{25-27}$ (Note also that there is no sharp distinction between impurity band double-exchange and kineticexchange interactions; the former is simply a strong coupling, narrow band limit of the latter.) A factor of 2 smaller $T_{c}$ in $(\mathrm{Ga}, \mathrm{Mn}) \mathrm{P}$, compared to $(\mathrm{Ga}, \mathrm{Mn}) \mathrm{As}$ prepared under similar growth conditions, ${ }^{25}$ indicates a marked suppression of the Curie temperature in this material due to the shorter range of magnetic interactions in the hopping regime.

The theoretical work presented in this paper is based on the currently best understood and highest $T_{c}$ (Ga,Mn)As benchmark material and explores whether Curie temperature can still be increased by introducing elements from the higher rows in the Periodic Table. In particular, we focus on magnetic and structural properties of Mn-doped $\mathrm{Ga}(\mathrm{As}, \mathrm{P})$ and $(\mathrm{Al}, \mathrm{Ga})$ As ternary hosts. We exploit a special circumstance that AlAs and GaAs lattice constants are very similar but GaP has a substantially smaller lattice constant, and that AlAs and $\mathrm{GaP}$ have very similar band offsets relative to the smaller gap GaAs (see Fig. 1). This allows us to disentangle the roles in the effective magnetic interaction between Mn and hole spins of the positions of Mn derived states relative to the valence-band edge, and of the overlaps between anion (As or P) $p$ orbitals forming the top of the valence band and the $\mathrm{Mn} d$ orbitals. Magnetic interactions are found to be more sensitive to the latter parameter. It explains why alloying (Ga,Mn)As with $\mathrm{Al}$ has only a small effect on Curie temperature $^{28}$ but we predict a sizable enhancement of the Curie temperature in the smaller lattice constant $\mathrm{Ga}(\mathrm{As}, \mathrm{P})$ ternary host. The mean-field $T_{c}$ calculations are presented in Sec. II together with estimates of the range of magnetic interactions in different $(\mathrm{Ga}, \mathrm{Mn})(\mathrm{As}, \mathrm{P})$ mixed crystals.

Structural properties of Mn-doped $\mathrm{Ga}(\mathrm{As}, \mathrm{P})$ and (Al,Ga)As ferromagnetic semiconductors are studied in Sec. III. Under equilibrium growth conditions the incorporation of $\mathrm{Mn}$ ions into $\mathrm{GaAs}$ is limited to less than $1 \%$. To circumvent the solubility problem a nonequilibrium, lowtemperature molecular-beam-epitaxy (LT-MBE) technique has to be used to achieve Mn doping concentrations larger than $1 \%$ at which ferromagnetism occurs. The highly doped LT-MBE (Ga,Mn)As systems show a strong tendency to selfcompensation by interstitial $\mathrm{Mn}_{\mathrm{I}}$ impurities. ${ }^{22,29,30}$ Correlations between acceptors $\left(\mathrm{Mn}_{\mathrm{Ga}}\right)$ and donors $\left(\mathrm{Mn}_{\mathrm{I}}\right)$ in $\mathrm{GaAs}^{22,31}$ are strong due to the nearly covalent nature of bonding in the crystal. The cohesion energy of the covalent networks has a maximum if the Fermi energy $E_{F}$ lies within the band gap. Whenever $E_{F}$ is shifted to the valence band the strength of the bonds is reduced because of the occurrence of unfilled bonding states. The tendency to self-compensation by $\mathrm{Mn}_{\mathrm{I}}$ can therefore be expected to weaken in wider gap hosts in which the substitutional Mn acceptor level is shifted deeper in the band gap. Another factor determining the formation energy of $\mathrm{Mn}_{\mathrm{I}}$ is the size of the interstitial space in the lattice it fits in, i.e., the host lattice constant. In Sec. III we again use the comparison between Mn-doped Ga(As,P) and (Al,Ga)As hosts, with similar positions of the substitutional Mn acceptor levels ${ }^{32,33}$ (see also Fig. 1) but different lattice constants, to separate the roles of the two factors. A significant decrease of the $\mathrm{Mn}_{\mathrm{I}}$ partial concentration observed only in $\mathrm{Ga}(\mathrm{As}, \mathrm{P})$ indicates that the lattice parameter plays a more important role than the position of the acceptor level in these mixed (III,Mn) V crystals.

We conclude our paper in Sec. IV with a brief discussion of the theoretical results which we believe provide motivation for a systematic experimental exploration of epitaxial $(\mathrm{Ga}, \mathrm{Mn})(\mathrm{As}, \mathrm{P})$ ferromagnetic semiconductor compounds.

\section{MAGNETIC INTERACTIONS}

The discussion in this section is divided into three parts. First we assume the Schrieffer-Wolf transformation of the many-body Anderson Hamiltonian and estimate trends in mean-field $T_{c}$ in $(\mathrm{Ga}, \mathrm{Mn}) \mathrm{As},(\mathrm{Ga}, \mathrm{Mn}) \mathrm{P}$, and (Al,Mn)As based on the effective interaction energy between local Mn moments and hole spins. In the second part we calculate band structures of Mn-doped $\mathrm{Ga}(\mathrm{As}, \mathrm{P})$ and $(\mathrm{Al}, \mathrm{Ga}) \mathrm{As}$ ternary hosts, using the microscopic tight-binding coherentpotential approximations (TBA/CPA), and calculate corresponding mean-field Curie temperatures. Results of the $a b$ initio $\mathrm{LDA}+\mathrm{U}$ calculations of the mean-field $T_{c}$ in $(\mathrm{Ga}, \mathrm{Mn})(\mathrm{As}, \mathrm{P})$ and of the range of hole mediated $\mathrm{Mn}-\mathrm{Mn}$ magnetic coupling are presented in the last subsection. The latter quantity is used to estimate the potential suppression of ferromagnetic interactions due to stronger binding of the hole to $\mathrm{Mn}$ in the wider gap host.

\section{A. Qualitative estimates of the kinetic-exchange coupling and mean-field $T_{c}$}

Effects of strong Coulomb correlations in the Mn $3 d$ shell and hybridization with the host semiconductor band states, which are at the heart of magnetism in these systems, can be qualitatively captured by the Anderson Hamiltonian. ${ }^{34-36}$ Here Coulomb correlations are modeled by the on-site Hubbard potential which depends on the occupation number of the $d$ states. The change in this effective potential when the number of occupied localized orbitals changes by one is parameterized by the Hubbard constant $U$. The localized orbital part of the Anderson Hamiltonian has an additional parameter, the Hunds rule constant $J_{H}$. This parameter captures the local direct exchange physics which favors spin-polarized open shell atomic states. For the case of the Mn- $d^{5}$ conguration, $J_{H}$ forces all five singly occupied $d$ orbitals to align their spins in the ground state.

The Schrieffer-Wolff transformation of the Anderson model removes the $p$ - $d$ hybridization term in the many body Hamiltonian and leads to a description in which localized Mn $d$ states interact with the valence band via a spin-spin interaction only. ${ }^{37}$ Near the $\Gamma$ point, the strength of this kinetic-exchange interaction can be parametrized by a constant

$$
J_{\text {pd }} \propto \Omega_{\text {u.c. }}\left|V_{\text {pd }}\right|^{2}\left(1 /\left|E_{d \uparrow}\right|+1 /\left|E_{d \downarrow}\right|\right),
$$


where $\Omega_{\text {u.c. }}=a_{\mathrm{lc}}^{3} / 4$ is the volume of the unit cell of the zincblende crystal with a lattice constant $a_{\mathrm{lc}}, V_{p d} \propto a_{\mathrm{lc}}^{-7 / 2}$ represents the hybridization potential, ${ }^{38}$ and $\left|E_{d \uparrow}\right|$ and $\left|E_{d \downarrow}\right|$ are the distances of the occupied and empty atomic Mn $d$ levels from $E_{F}\left(\left|E_{d \downarrow}-E_{d \uparrow}\right|=U+5 J_{H}\right) \cdot 37,39,40$

In the mean-field kinetic-exchange model the Curie temperature of a $\mathrm{III}_{1-x} \mathrm{Mn}_{x} \mathrm{~V}$ magnetic semiconductor scales as ${ }^{6,7}$ $T_{c} \propto J_{\mathrm{pd}}^{2} x / \Omega_{\text {u.c. }}$, i.e.,

$$
T_{c} \propto a_{\mathrm{lc}}^{-11}\left(1 /\left|E_{d \uparrow}\right|+1 /\left|E_{d \downarrow}\right|\right)^{2} .
$$

The effect of the second term in Eq. (2) on $T_{c}$ in GaP or AlAs hosts, compared to GaAs, can be estimated from Fig. 1. Despite the twice as large band gap, the valence band offset is relatively small and the increase of $1 /\left|E_{d \uparrow}\right|$ is almost completely compensated by the decrease of $1 /\left|E_{d \downarrow}\right|$. The second term in Eq. (2) therefore leads to only a $4 \%$ enhancement of the mean-field kinetic-exchange model $T_{c}$ in both $\mathrm{GaP}$ and AlAs hosts as compared to GaAs.

A much stronger enhancement of $T_{c}$ is obtained for $\mathrm{GaP}$ due to the first term in Eq. (2). Considering $a_{\mathrm{lc}}=5.653 \AA$ for GaAs and $a_{\mathrm{lc}}=5.450 \AA$ for $\mathrm{GaP}^{41}$ the mean-field kineticexchange model $T_{c}$ is by $50 \%$ larger in $(\mathrm{Ga}, \mathrm{Mn}) \mathrm{P}$ than in $(\mathrm{Ga}, \mathrm{Mn})$ As. For AlAs, on the other hand, no marked change in $T_{c}$ is expected from Eq. (2) since the material is nearly lattice matched with GaAs $\left(a_{\mathrm{lc}}=5.661 \AA\right.$, for AlAs).

\section{B. Microscopic tight-binding model calculations}

An effective single-particle TBA band structure can be obtained from the Anderson Hamiltonian by replacing the density operators in the Hubbard terms with their mean values. ${ }^{39}$ We use this approach here, combined with the CPA, to calculate microscopically mean-field Curie temperatures in Mn-doped $\mathrm{Ga}(\mathrm{As}, \mathrm{P})$ and $(\mathrm{Al}, \mathrm{Ga}) \mathrm{As}$ mixed crystals. Our TBA Hamiltonian includes the $8 \times 8 s p^{3}$ term with secondneighbor-interaction integrals describing the host semiconductor $^{42}$ and terms describing hybridization with Mn. Local changes of the crystal potential at $\mathrm{Mn}$ are represented by shifted atomic levels. The parameters chosen for the atomic level shifts and the hopping amplitudes between atoms ${ }^{39,42}$ provide the correct band gap for the binary host crystal and the appropriate exchange splitting of the Mn $d$ states. In particular, we considered in the TBA/CPA calculations $U$ $=3.5 \mathrm{eV}$ and $J_{H}=0.6 \mathrm{eV}$, resulting in approximately $6 \mathrm{eV}$ splitting of the peaks of the majority and minority $\mathrm{Mn}$ $d$-projected density of states in $(\mathrm{Ga}, \mathrm{Mn}) \mathrm{As}$. For the ternary $\mathrm{GaP}_{y} \mathrm{As}_{1-y}$ and $\mathrm{Al}_{y} \mathrm{Ga}_{1-y} \mathrm{As}$ mixed crystals we used the energy scale related to the GaAs band structure and shifted the atomic level of the other components according to the band offsets of binary hosts shown in Fig. 1. The nondiagonal matrix elements of the TBA Hamiltonian appropriate for the mixed crystals were obtained by linear interpolation applied to the hopping integrals multiplied by the lattice constant squared, ${ }^{38}$

$$
V(y)=\frac{y V(1) a_{\mathrm{lc}}^{2}(1)+(1-y) V(0) a_{\mathrm{lc}}^{2}(0)}{\left[y a_{\mathrm{lc}}(1)+(1-y) a_{\mathrm{lc}}(0)\right]^{2}} .
$$

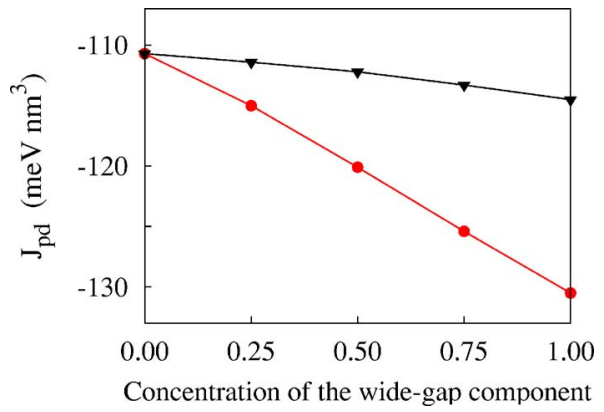

FIG. 2. (Color online) TBA/CPA $J_{\text {pd }}$ in $\mathrm{Al}_{y} \mathrm{Ga}_{1-y} \mathrm{As}$ (black triangles) and $\mathrm{GaP}_{y} \mathrm{As}_{1-y}$ (red dots) hosts doped with $x=10 \% \mathrm{Mn}$.

We used the same values of $U$ and $J_{H}$ for all mixed crystals appealing to the nearly atomiclike, $d^{5}$ character of $\mathrm{Mn}$ impurity atoms.

Within the CPA, disorder effects associated with random distribution of $\mathrm{Mn}_{\text {III }}$ and of $\mathrm{Al}_{\mathrm{Ga}}$ or $\mathrm{P}_{\mathrm{As}}$ appear in the finite spectral width of hole quasiparticle states. Since realizations with near-neighbor Mn ions are included within the disorderaveraged TBA/CPA with the proper statistical probability, short-range local moment interactions (such as antiferromagnetic superexchange) contribute to the final magnetic state. In uncompensated systems considered here, however, the long-range ferromagnetic $\mathrm{Mn}-\mathrm{Mn}$ coupling mediated by holes dominates.

In Fig. 2 we plot the dependence of the kinetic-exchange constant $J_{\text {pd }}$, derived from the splitting of the TBA/CPA valence band at the $\Gamma$ point, as a function of the concentration of the wide-gap component $y$. As expected from the qualitative discussion above, $J_{\mathrm{pd}}$ depends only weakly on the concentration of $\mathrm{Al}$ in the $\mathrm{Al}_{y} \mathrm{Ga}_{1-y} \mathrm{As}$ host. A stronger variation is obtained for $\mathrm{GaP}_{y} \mathrm{As}_{1-y}$ with $J_{\mathrm{pd}}$ enhancement of $16 \%$ at $y=1$, in good agreement with Eq. (1).

The TBA/CPA Curie temperatures are obtained using the compatibility of the model with the Weiss mean-field theory. The strength of the Mn-Mn coupling is characterized by the energy cost, $E_{\mathrm{rev}}$, of flipping one Mn moment, which can be calculated for a given chemical composition. ${ }^{39} E_{\mathrm{rev}}$ is proportional to the effective Weiss field and to the mean-field Curie temperature, $k_{B} T_{c}=E_{\text {rev }} / 6$. In Fig. 3 we plot the TBA/CPA $T_{c}$ in the $\mathrm{GaP}_{y} \mathrm{As}_{1-y}$ host for $5 \%$ and $10 \% \mathrm{Mn}$ concentrations. A factor of 1.5-2 enhancement of $T_{c}$ for $y=1$ compared to the

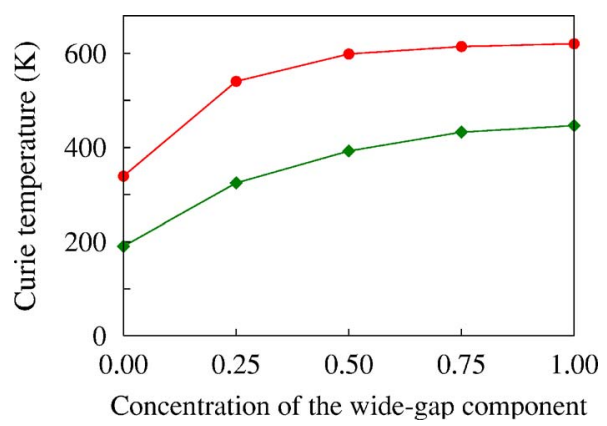

FIG. 3. (Color online) TBA/CPA mean-field $T_{c}$ in $\mathrm{GaP}_{y} \mathrm{As}_{1-y}$ hosts doped with $x=5 \% \mathrm{Mn}$ (green diamonds) and 10\% Mn (red dots). 


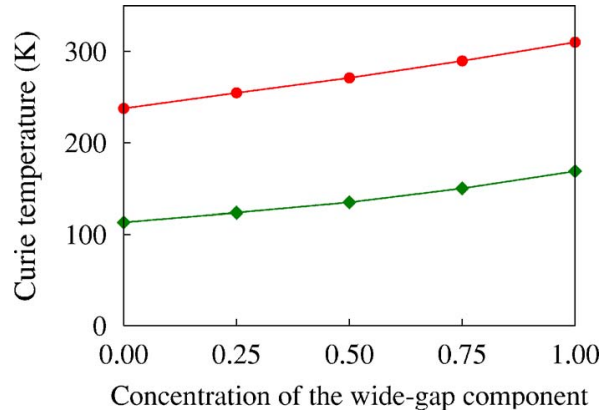

FIG. 4. (Color online) $A b$ initio $\mathrm{LDA}+\mathrm{U}$ mean-field $T_{c}$ in $\mathrm{GaP}_{y} \mathrm{As}_{1-y}$ hosts doped with $x=5 \% \mathrm{Mn}$ (green diamonds) and $10 \%$ Mn (red dots).

GaAs host is consistent with qualitative predictions in the preceding section. Note that in $(\mathrm{Al}, \mathrm{Ga})$ As hosts, the microscopic TBA/CPA $T_{c}$ shows only weak dependence on the Al content, again consistent with the discussion in the preceding section and with Fig. 2.

\section{C. $A b$ initio $\mathrm{LDA}+\mathrm{U}$ theory}

In ab initio approaches, the CPA description of disordered mixed crystals is combined with the local density approximation (LDA) to density-functional theory. Electron correlations on the Mn $d$ shell are modeled by including Hubbard interaction terms in the Hamiltonian $(\mathrm{LDA}+\mathrm{U}) .{ }^{43-45}$ The $\mathrm{LDA}+\mathrm{U} / \mathrm{CPA}$ method is implemented within the framework of the first-principles, tight-binding linear muffin-tin orbital approach. ${ }^{46,47}$ Hubbard parameters used in the ab initio calculations were chosen to provide similar exchange splitting of the Mn $d$ states as obtained in the TBA/CPA model calculations.

The mean field $T_{c}$ 's, shown in Fig. 4, are obtained again from the spin-flip energy $E_{\text {rev }}$ which is calculated directly from the LDA+U/CPA Green's functions. ${ }^{48} T_{c}$ clearly increases with increasing $y$ in the series of $\mathrm{Ga}_{1-x} \mathrm{Mn}_{x} \mathrm{As}_{1-y} \mathrm{P}_{y}$ with fixed Mn doping $x$. Also in agreement with model calculations of previous sections, the LDA +U/CPA values of the mean-field $T_{c}$ are proportional to the Mn concentration within the studied range of $\mathrm{Mn}$ dopings of 1-10\%. Note that while the main Curie temperature trends with Mn and P doping are described consistently by the different theoretical approaches employed in this paper the absolute values of $T_{c}$ 's cannot be predicted with a high quantitative accuracy. This is typical for microscopic theories of dilute moment ferromagnetic semiconductors. ${ }^{1}$ In the case of TBA/CPA and LDA $+\mathrm{U} / \mathrm{CPA}$ techniques we attribute the discrepancy to significantly smaller band gaps in the LDA+U/CPA spectra. For GaAs, e.g., the LDA+U band gap is $0.4 \mathrm{meV}$, compared to the TBA (and experimental) band gap of $1.5 \mathrm{eV}$. The valence band edge in the LDA+U spectra is then shifted further from the majority Mn $d$ level and the $p$ - $d$ hybridization is suppressed, resulting in smaller mean-field $T_{c}$ values.

The energy $E_{\text {rev }}$ could also be obtained from the interatomic exchange parameters $J_{i j}$ constructed by mapping the LDA+U/CPA total energy on the Heisenberg Hamiltonian: ${ }^{47,48}$

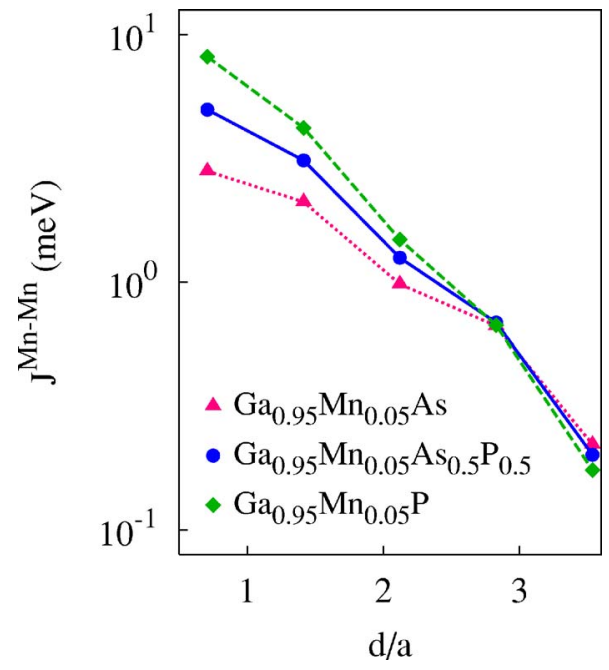

FIG. 5. (Color online) Ab initio LDA $+\mathrm{U}$ range of $\mathrm{Mn}-\mathrm{Mn}$ interactions in $\mathrm{GaP}_{y} \mathrm{As}_{1-y}$ hosts with $y=0,0.5$, and 1 along [110] crystal direction.

$$
H=-\sum_{i j} J_{i j} \hat{e}_{i} \cdot \hat{e}_{j}
$$

where $\hat{e}_{i}$ is the local moment unit vector. Since the moments induced on nonmagnetic atom sites are small, the summation can be restricted to sites occupied by Mn and

$$
E_{\mathrm{rev}}=2 \sum_{j} J_{i j} \approx 2 x \sum_{j} J_{i j}^{\mathrm{Mn}-\mathrm{Mn}} .
$$

Individual $J_{i j}$ potentials characterize the spatial extent of the $\mathrm{Mn}-\mathrm{Mn}$ exchange coupling. This parameter is particularly useful for estimating corrections beyond mean-field theory in dilute moment systems. The mean-field approximation is more reliable for sufficiently long-range character of carrier mediated Mn-Mn coupling but tends to overestimate $T_{c}$ when the carriers become more localized and magnetic interactions short ranged. ${ }^{49-51}$ As shown in Fig. 5, the range of $\mathrm{Mn}-\mathrm{Mn}$ exchange interactions is similar within the whole family of $\mathrm{Ga}(\mathrm{As}, \mathrm{P})$ ternary hosts and safely exceeds the average $\mathrm{Mn}-\mathrm{Mn}$ moment distance. We therefore expect that results of mean-field theory reliably describe qualitative $T_{c}$ trends in $\mathrm{Mn}$ doped $\mathrm{Ga}(\mathrm{As}, \mathrm{P})$ mixed crystals.

\section{PARTIAL CONCENTRATION OF INTERSTITIAL Mn}

In the preceding section we have considered all Mn impurities to substitute for the group-III element. In Mn-doped GaAs materials, however, a fraction of $\mathrm{Mn}$ is incorporated during the growth at interstitial positions. These donor impurities are likely to form pairs with substitutional Mn acceptors in as-grown systems with approximately zero net moment, ${ }^{52-54}$ resulting in an effective free local-moment doping $x_{\text {eff }}=x_{s}-x_{i}$. Here $x_{s}$ and $x_{i}$ are partial concentrations of substitutional and interstitial Mn, respectively. Although $\mathrm{Mn}_{\mathrm{I}}$ can be removed by low-temperature annealing, $x_{\text {eff }}$ will remain smaller than the total nominal Mn doping, $x=x_{s}+x_{i}$. The substitutional Mn doping efficiency is, therefore, one of 


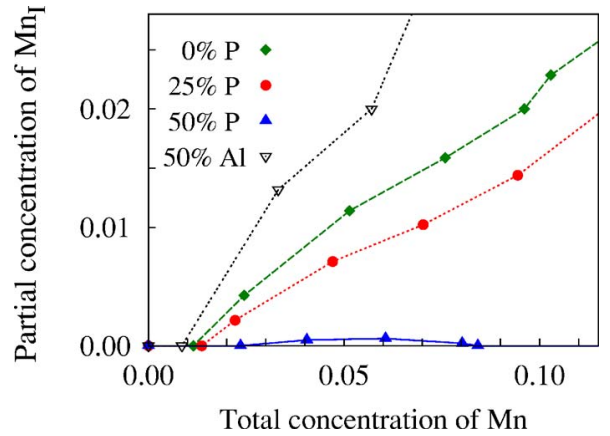

FIG. 6. (Color online) Partial concentration of $\mathrm{Mn}_{\mathrm{I}}$ as a function of total $\mathrm{Mn}$ concentration in $\mathrm{GaP}_{y} \mathrm{As}_{1-y}$ with $y=0,0.25$, and 0.5 , and $\mathrm{Al}_{y} \mathrm{Ga}_{1-y} \mathrm{As}$ with $y=0.5$.

the key parameters that may limit $T_{c}$ also in the wider gap, $p$-type III-V hosts.

As discussed in the Introduction, the increasing ionicity and energy of the substitutional Mn acceptor level with increasing concentration of the wider gap element is one factor that may reduce the tendency to self-compansation by $\mathrm{Mn}_{\mathrm{I}}$. This mechanism should play a comparable role in both $\mathrm{Ga}(\mathrm{As}, \mathrm{P})$ and $(\mathrm{Al}, \mathrm{Ga})$ As hosts, given the similar experimental positions of the $\mathrm{Mn}$ acceptor level in AlAs, $E_{a}$ $\approx 0.44 \mathrm{eV},{ }^{32}$ and in $\mathrm{GaP}, E_{a} \approx 0.4 \mathrm{eV} .{ }^{33}$ The formation energy of $\mathrm{Mn}_{\mathrm{I}}$ may, however, also increase due to a geometrical effect of a reduced size of the interstitial space in smaller lattice constant hosts. Calculations for $\mathrm{Ga}(\mathrm{As}, \mathrm{P})$ and for the nearly GaAs lattice matched (Al,Ga)As, shown in Fig. 6, indicate that the geometrical effect dominates.

The compositional dependence of the $\mathrm{Mn}_{\mathrm{I}}$ partial concentration, plotted in Fig. 6, is obtained from ab initio formation energies of substitutional and interstitial $\mathrm{Mn}^{22,31,55}$ calculated for the given $\mathrm{GaP}_{y} \mathrm{As}_{1-y}$ or $\mathrm{Al}_{y} \mathrm{Ga}_{1-y} \mathrm{As}$ host. In the smaller lattice constant $\mathrm{Ga}(\mathrm{As}, \mathrm{P})$, the formation energy of $\mathrm{Mn}_{\mathrm{I}}$ is enhanced and the concentration of $\mathrm{Mn}_{\mathrm{I}}$ is significantly suppressed already at $y=0.25$ and no $\mathrm{Mn}_{\mathrm{I}}$ impurities are expected to form at $y>0.5$. If confirmed experimentally, this property might have a profound effect on both structural and magnetic quality of Mn-doped $\mathrm{Ga}(\mathrm{As}, \mathrm{P})$ epilayers.

In the lattice-matched $(\mathrm{Al}, \mathrm{Ga}) \mathrm{As}$, the formation energy of $\mathrm{Mn}_{\mathrm{I}}$ is independent of the $\mathrm{Al}$ concentration and, correspondingly, our calculations show no suppression of $\mathrm{Mn}_{\mathrm{I}}$ formation in this ternary host. Note that the theoretical results in Fig. 6 predict even a marked increase of the $\mathrm{Mn}_{\mathrm{I}}$ partial concentration due to Al. This behavior reflects the high formation energy of substitutional $\mathrm{Mn}_{\mathrm{Al}}$ impurities and may eventually change the type of conduction and ferromagnetism in $\mathrm{Mn}$ doped $(\mathrm{Al}, \mathrm{Ga}) \mathrm{As}$ with large $\mathrm{Al}$ concentrations. A detailed microscopic study of systems whose properties are dominated by interstitial Mn impurities is beyond the scope of this paper and will be discussed elsewhere.

\section{DISCUSSION}

The effect of alloying (Ga,Mn)As with P on Curie temperature has been discussed theoretically in a previous study by $\mathrm{Xu}$ et al. ${ }^{17}$ Assuming the double-exchange model for the entire family of Mn-doped $\mathrm{Ga}(\mathrm{As}, \mathrm{P})$ hosts the authors found no significant enhancement of $T_{c}$ with increasing $\mathrm{P}$ content. In $a b$ initio theories the character of magnetic interactions for a given (III,Mn) $\mathrm{V}$ dilute moment system shifts from the delocalized-hole kinetic-exchange towards the impurity-band double exchange when removing the Hubbard correlation potentials. Indeed, in agreement with $\mathrm{Xu}$ et al., we found only weak dependence of the mean-field $T_{c}$ on the As/P ratio when replacing the LDA+U/CPA band structure with energy spectra obtained using pure LDA/CPA. Extensive experimental studies of MBE-grown $(\mathrm{Ga}, \mathrm{Mn}) \mathrm{As}$ favor the kinetic-exchange model while data measured in the Mn ionimplanted GaP material are more readily interpreted within the double-exchange model. ${ }^{1}$ This may suggest that theoretical $T_{c}$ trends in Figs. 3 and 4 apply to $\mathrm{Ga}(\mathrm{As}, \mathrm{P})$ hosts with lower $\mathrm{P}$ content while the results of $\mathrm{Xu}$ et al. (or the LDA/ CPA theory) describe more reliably the opposite P-doping limit. The range of validity of either of the theoretical pictures can be ultimately established only by a detailed study of epitaxial $(\mathrm{Ga}, \mathrm{Mn})(\mathrm{As}, \mathrm{P})$ compounds with variable $\mathrm{Mn}$ and $\mathrm{P}$ concentrations and minimal number of other unintentional impurities or lattice defects.

For practical reasons phosphorus cells are rarely mounted on laboratory (III,Mn)-V MBE systems. ${ }^{56,57}$ Note, however, that useful complementary studies of hole localization effects on magnetic interactions in ternary hosts derived from GaAs can be performed by alloying with the much more common Al. The tendency to localization and reduced range of Mn-Mn exchange interactions should be comparable in $(\mathrm{Al}, \mathrm{Ga}) \mathrm{As}$ and $\mathrm{Ga}(\mathrm{As}, \mathrm{P})$, given the similar position of the $\mathrm{Mn}$ acceptor level in these two hosts ${ }^{32,33}$ (see Fig. 1). Experimental results ${ }^{28}$ in annealed, 5\% Mn-doped $\mathrm{Al}_{y} \mathrm{Ga}_{1-y} \mathrm{As}$ materials grown by LT-MBE with $y$ ranging from 0 to 0.3 showed no marked dependence of $T_{c}$ on $y$. For the $(\mathrm{Al}, \mathrm{Ga}) \mathrm{As}$ ternary hosts which are lattice matched to GaAs and for which the kinetic-exchange picture implies $T_{c}$ 's nearly independent of $\mathrm{Al}$ concentration, this experimental result can be interpreted as a signature of comparably longranged $\mathrm{Mn}-\mathrm{Mn}$ interactions in the studied $(\mathrm{Al}, \mathrm{Ga}) \mathrm{As}$ hosts as in the pure GaAs host. According to Figs. 1-4 it also implies, however, that $T_{c}$ should increase when alloying $(\mathrm{Ga}, \mathrm{Mn}) \mathrm{As}$ with P.

To conclude, we found two motivating factors in the materials research of high temperature diluted ferromagnetic semiconductors for performing a detailed experimental study of epitaxial $(\mathrm{Ga}, \mathrm{Mn})(\mathrm{As}, \mathrm{P})$ : We expect the Curie temperature to increase with increasing $\mathrm{P}$ concentration with an enhancement factor of up to $\sim 1.5$ compared to $(\mathrm{Ga}, \mathrm{Mn}) \mathrm{As}$ with the same concentration of uncompensated $\mathrm{Mn}_{\mathrm{Ga}}$ local moments. We also predict a significantly weaker tendency to carrier and moment self-compenstion by interstitial $\mathrm{Mn}_{\mathrm{I}}$ impurities in $\mathrm{Ga}(\mathrm{As}, \mathrm{P})$ hosts compared to pure GaAs. Finally, we point out that Mn-doped $(\mathrm{Al}, \mathrm{Ga}) \mathrm{As}$ represents a useful complementary system to $(\mathrm{Ga}, \mathrm{Mn})(\mathrm{As}, \mathrm{P})$ for understanding trends in magnetic and structural properties of these wider gap III-V hosts.

\section{ACKNOWLEDGMENTS}

We acknowledge fruitful discussions with Tom Foxon, 
Andrew Rushforth, and Alexander Shick, and support from the Grant Agency of the Czech Republic under Grant No. 202/05/0575 and 202/04/0583, from the Academy of Sciences of the Czech Republic under Institutional Support No. AVOZ10100521 and AVOZ10100520, from the Ministry of Education of the Czech Republic Center for Fundamental
Research LC510 and COST P19 OC-150, from the UK EPSRC under Grant No. GR/S81407/01, from the National Science Foundation under Grant No. PHY99-07949, from NSF Grant No. DMR-0547875, and from ONR Grant No. ONR-N000140610122. Jairo Sinova is a Cottrell Scholar of Research Corporation.
${ }^{1}$ T. Jungwirth, J. Sinova, J. Mašek, J. Kučera, and A. H. MacDonald, Rev. Mod. Phys. 78, 809 (2006).

${ }^{2}$ T. Schulthess, W. M. Temmerman, Z. Szotek, W. H. Butler, and G. M. Stocks, Nat. Mater. 4, 838 (2005).

${ }^{3}$ P. M. Krstajić, F. M. Peeters, V. A. Ivanov, V. Fleurov, and K. Kikoin, Phys. Rev. B 70, 195215 (2004).

${ }^{4}$ T. Dietl, A. Haury, and Y. M. d'Aubigne, Phys. Rev. B 55, R3347 (1997).

${ }^{5}$ F. Matsukura, H. Ohno, A. Shen, and Y. Sugawara, Phys. Rev. B 57, R2037 (1998).

${ }^{6}$ T. Jungwirth, W. A. Atkinson, B. H. Lee, and A. H. MacDonald, Phys. Rev. B 59, 9818 (1999).

${ }^{7}$ T. Dietl, H. Ohno, F. Matsukura, J. Cibert, and D. Ferrand, Science 287, 1019 (2000).

${ }^{8}$ M. Jain, L. Kronik, J. R. Chelikowsky, and V. V. Godlevsky, Phys. Rev. B 64, 245205 (2001).

${ }^{9}$ J. Inoue, S. Nonoyama, and H. Itoh, Phys. Rev. Lett. 85, 4610 (2000).

${ }^{10}$ V. I. Litvinov and V. K. Dugaev, Phys. Rev. Lett. 86, 5593 (2001).

${ }^{11}$ A. C. Durst, R. N. Bhatt, and P. A. Wolff, Phys. Rev. B 65 , 235205 (2002).

12 A. L. Chudnovskiy and D. Pfannkuche, Phys. Rev. B 65, 165216 (2002).

${ }^{13}$ A. Kaminski and S. Das Sarma, Phys. Rev. Lett. 88, 247202 (2002).

${ }^{14}$ M. Berciu and R. N. Bhatt, Phys. Rev. Lett. 87, 107203 (2001).

${ }^{15}$ R. N. Bhatt, M. Berciu, M. P. Kennett, and X. Wan, J. Supercond. 15, 71 (2002).

${ }^{16}$ G. Alvarez, M. Mayr, and E. Dagotto, Phys. Rev. Lett. 89, 277202 (2002).

${ }^{17}$ J. L. Xu and M. van Schilfgaarde, J. Magn. Magn. Mater. 305, 63 (2006).

${ }^{18}$ J. M. Langer, C. Delerue, M. Lannoo, and H. Heinrich, Phys. Rev. B 38, 7723 (1988).

${ }^{19}$ T. Dietl, Semicond. Sci. Technol. 17, 377 (2002).

${ }^{20}$ M. Csontos, G. Mihály, B. Jankó, T. Wojtowicz, X. Liu, and J. K. Furdyna, Nat. Mater. 4, 447 (2005).

${ }^{21}$ K. Y. Wang, R. P. Campion, K. W. Edmonds, M. Sawicki, T. Dietl, C. T. Foxon, and B. L. Gallagher, in Proceedings of the 27th International Conference on the Physics of Semiconductors, edited by J. M. C. G. V. de Walle, AIP Conf. Proc. No. 772 (AIP, Melville, NY, 2005), p. 333, cond-mat/0411475.

${ }^{22}$ T. Jungwirth et al., Phys. Rev. B 72, 165204 (2005).

${ }^{23}$ T. Graf, M. Gjukic, M. S. Brandt, M. Stutzmann, and O. Ambacher, Appl. Phys. Lett. 81, 5159 (2002).

${ }^{24}$ J. I. Hwang et al., Phys. Rev. B 72, 085216 (2005).

${ }^{25}$ M. A. Scarpulla, B. L. Cardozo, R. Farshchi, W. M. Hlaing Oo, M. D. McCluskey, K. M. Yu, and O. D. Dubon, Phys. Rev. Lett.
95, 207204 (2005).

${ }^{26}$ N. Theodoropoulou, A. F. Hebard, M. E. Overberg, C. R. Abernathy, S. J. Pearton, S. N. G. Chu, and R. G. Wilson, Phys. Rev. Lett. 89, 107203 (2002).

${ }^{27}$ P. Poddar, Y. Sahoo, H. Srikanth, and P. N. Prasad, Appl. Phys. Lett. 87, 062506 (2005).

${ }^{28}$ K. Takamura, F. Matsukura, D. Chiba, and H. Ohno, Appl. Phys. Lett. 81, 2590 (2002).

${ }^{29}$ K. M. Yu, W. Walukiewicz, T. Wojtowicz, I. Kuryliszyn, X. Liu, Y. Sasaki, and J. K. Furdyna, Phys. Rev. B 65, 201303(R) (2002).

${ }^{30}$ F. Máca and J. Mašek, Phys. Rev. B 65, 235209 (2002).

${ }^{31}$ J. Mašek, I. Turek, J. Kudrnovský, F. Máca, and V. Drchal, Acta Phys. Pol. A 105, 637 (2004).

${ }^{32}$ F. Bantien and J. Weber, Phys. Rev. B 37, 10111 (1988).

${ }^{33}$ B. Clerjaud, J. Phys. C 18, 3615 (1985).

${ }^{34}$ P. W. Anderson, Phys. Rev. 124, 41 (1961).

${ }^{35}$ F. D. M. Haldane and P. W. Anderson, Phys. Rev. B 13, 2553 (1976).

${ }^{36}$ V. N. Fleurov and K. A. Kikoin, J. Phys. C 9, 1673 (1976).

${ }^{37}$ J. R. Schrieffer and P. A. Wolff, Phys. Rev. 149, 491 (1966).

${ }^{38} \mathrm{~W}$. Harrison, Electronic Structure and the Properties of Solid (Freeman, San Francisco, 1980).

${ }^{39}$ J. Mašek, Solid State Commun. 78, 351 (1991).

${ }^{40}$ C. Timm and A. H. MacDonald, Phys. Rev. B 71, 155206 (2005).

${ }^{41}$ I. Vurgaftman, J. R. Meyer, and L. R. Ram-Mohan, J. Appl. Phys. 89, 5815 (2001).

${ }^{42}$ D. N. Talwar and C. S. Ting, Phys. Rev. B 25, 2660 (1982).

${ }^{43}$ J. H. Park, S. K. Kwon, and B. I. Min, Physica B 281/282, 703 (2000).

${ }^{44}$ A. B. Shick, J. Kudrnovský, and V. Drchal, Phys. Rev. B 69, 125207 (2004).

${ }^{45}$ M. Wierzbowska, D. Sanchez-Portal, and S. Sanvito, Phys. Rev. B 70, 235209 (2004).

${ }^{46}$ I. Turek, V. Drchal, J. Kudrnovský, M. Šob, and P. Weinberger, Electronic Structure of Disordered Alloys, Surfaces, and Interfaces (Kluwer Academic, Boston, 1997).

${ }^{47}$ J. Kudrnovský, I. Turek, V. Drchal, F. Máca, P. Weinberger, and P. Bruno, Phys. Rev. B 69, 115208 (2004).

${ }^{48}$ A. I. Liechtenstein, M. I. Katsnelson, V. P. Antropov, and V. A. Gubanov, J. Magn. Magn. Mater. 67, 65 (1987).

${ }^{49}$ K. Sato, W. Schweika, P. H. Dederichs, and H. KatayamaYoshida, Phys. Rev. B 70, 201202(R) (2004).

${ }^{50}$ L. Bergqvist, O. Eriksson, J. Kudrnovský, V. Drchal, P. Korzhavyi, and I. Turek, Phys. Rev. Lett. 93, 137202 (2004).

${ }^{51}$ G. Bouzerar, T. Ziman, and J. Kudrnovský, Europhys. Lett. 69, 812 (2005)

${ }^{52}$ J. Blinowski and P. Kacman, Phys. Rev. B 67, 121204(R) (2003). 
${ }^{53}$ J. Mašek and F. Máca, Phys. Rev. B 69, 165212 (2003).

${ }^{54}$ K. W. Edmonds et al., Phys. Rev. Lett. 92, 037201 (2004).

${ }^{55}$ J. Mašek, I. Turek, V. Drchal, J. Kudrnovský, and F. Máca, Acta Phys. Pol. A 102, 673 (2002).
${ }^{56}$ M. E. Overberg, B. P. Gila, C. R. Abernathy, S. J. Pearton, N. A. Theodoropoulou, K. T. McCarthy, S. B. Arnason, and A. F. Hebard, Appl. Phys. Lett. 79, 3128 (2001).

${ }^{57}$ M. E. Overberg, J. Vac. Sci. Technol. B 20, 969 (2002). 